\title{
XMM-Newton observations of the dwarf elliptical galaxy NGC 3226
}

\author{
P. Gondoin, A. Orr, and H. Siddiqui
}

\author{
Research and Scientific Support Department, European Space Agency - Postbus 299, 2200 AG Noordwijk, The Netherlands
}

Received 9 December 2003 / Accepted 22 March 2004

\begin{abstract}
We report on an XMM-Newton observation of the dwarf elliptical galaxy NGC 3226 performed in November 2000. The analysis of the $0.4-10 \mathrm{keV}$ spectrum of its nucleus is consistent with a power law continuum $(\Gamma \approx 1.96)$ absorbed at low energies by neutral gas (with a hydrogen column density $N_{\mathrm{H}} \approx 3-8 \times 10^{21} \mathrm{~cm}^{-2}$ and a covering fraction greater than $85 \%$ ) and by weakly ionized gas $\left(N_{\mathrm{W}} \approx 4-6 \times 10^{21} \mathrm{~cm}^{-2}\right.$ with $\left.\xi \approx 1-7 \mathrm{erg} \mathrm{s}^{-1} \mathrm{~cm}\right)$. However, the study indicates that a bremstrahlung model absorbed by neutral material is a better description reminiscent of the X-ray emission from advection-dominated accretion flows (ADAFs). The temperature of the best fit bremstrahlung model $\left(T \approx 10^{8} \mathrm{~K}\right)$ and the absence of short and mid time-scale variability suggests that the $\mathrm{X}$-ray emission originates from regions relatively far from the nucleus as in convection-dominated accretion flows (CDAFs) or in the so-called "wind" models. By comparing the $2-10 \mathrm{keV}$ luminosity of the central object $\left(L_{\mathrm{X}}=2.58 \times 10^{40} \mathrm{erg} \mathrm{s}^{-1}\right.$ after correction from Galactic and intrinsic absorption) with radio flux measurements, we find a mass in the range $(1.7-50) \times 10^{7} M_{\odot}$ for the accreting black hole, a value comparable with an independent estimate from the dispersion of radial velocities. We argue that the dwarf elliptical galaxy NGC 3226 may harbor a sub-Eddington, super-massive black hole in a radiative inefficient state which is distinct from the near-Eddington, thermally dominated black holes presumably found in quasars and luminous Seyfert galaxies.
\end{abstract}

Key words. X-rays: individual: NGC 3226 - galaxies: nuclei - galaxies: elliptical and lenticular, cD - X-rays: galaxies

\section{Introduction}

NGC 3226 is a dwarf elliptical galaxy $(z=0.00441 \pm 0.00006$; de Vaucouleurs et al. 1991) which shows signs of interaction with the nearby SAB(s)a galaxy NGC 3227. Ho et al. (1997) found a moderately strong broad $\mathrm{H} \alpha$ component in the spectrum of the NGC 3226 nucleus. This result and the detection of compact, non-thermal radio emission from NGC 3226 (Falcke et al. 2000) are a good evidence for the presence of an active galactic nucleus (AGN) in the center of NGC 3226. The center of the galaxy was classified as a low ionization nuclear emission line region (LINER) of optical spectroscopic type 1.9 (Ho et al. 1997). X-ray emission from the NGC 3226 nucleus has been detected using the ROSAT Position Sensitive Proportional Counter (Sansom et al. 2000). The limited signal to noise ratio of the ROSAT data did not allow to distinguish between an AGN-like power law spectrum and a collisionally ionized plasma due to hot gas possibly from a starburst region. However, recent X-ray spectra obtained with the Chandra observatory support evidence from other spectral bands that the central region of NGC 3226 harbors a low luminosity active nucleus (George et al. 2001).

NGC 3227 was observed by the XMM-Newton space observatory in November 2000 (Gondoin et al. 2003). The 30' field of view of the EPIC cameras on board XMM-Newton included the NGC 3226 nucleus which was located $2^{\prime}$ off-axis from the observatory pointing direction towards the center of
NGC 3227. In the present paper, we report the analysis results of the X-ray emission from the central region of NGC 3226. Section 2 details the observations and data reduction procedures. Section 3 presents the integrated flux measurements and their temporal behavior during the observations. Section 4 describes the spectral analysis which was performed in two steps including first a characterization of the continuum emission above $3 \mathrm{keV}$ and then an analysis of the entire $0.3-10 \mathrm{keV}$ energy range. The results are discussed in Sect. 5.

\section{Observations and data reduction}

The data were obtained by the XMM-Newton space observatory (Jansen et al. 2001) between 2000 November 28, 18:10:23 (UT) and 2000 November 29, 05:25:18 (UT) for usable exposure times of $35.3 \mathrm{ks}$ (EPIC p-n camera) and $37.4 \mathrm{ks}$ (EPIC MOS 1 and MOS 2 cameras). XMM-Newton uses three grazing incidence telescopes which provide an effective area $>4000 \mathrm{~cm}^{2}$ at $2 \mathrm{keV}$ and $1600 \mathrm{~cm}^{2}$ at $8 \mathrm{keV}$ (Gondoin et al. 2000). Three EPIC CCD cameras (Strüder et al. 2001; Turner et al. 2001) at the prime focus of the telescopes provide imaging in a 30' field of view and broadband spectroscopy with a resolving power of between 5 and 60 in the energy range 0.3 to $10 \mathrm{keV}$.

The observations of NGC 3226 were conducted with the EPIC cameras operating in full frame mode. "Medium" thickness aluminum filters were used in front of all CCD cameras 

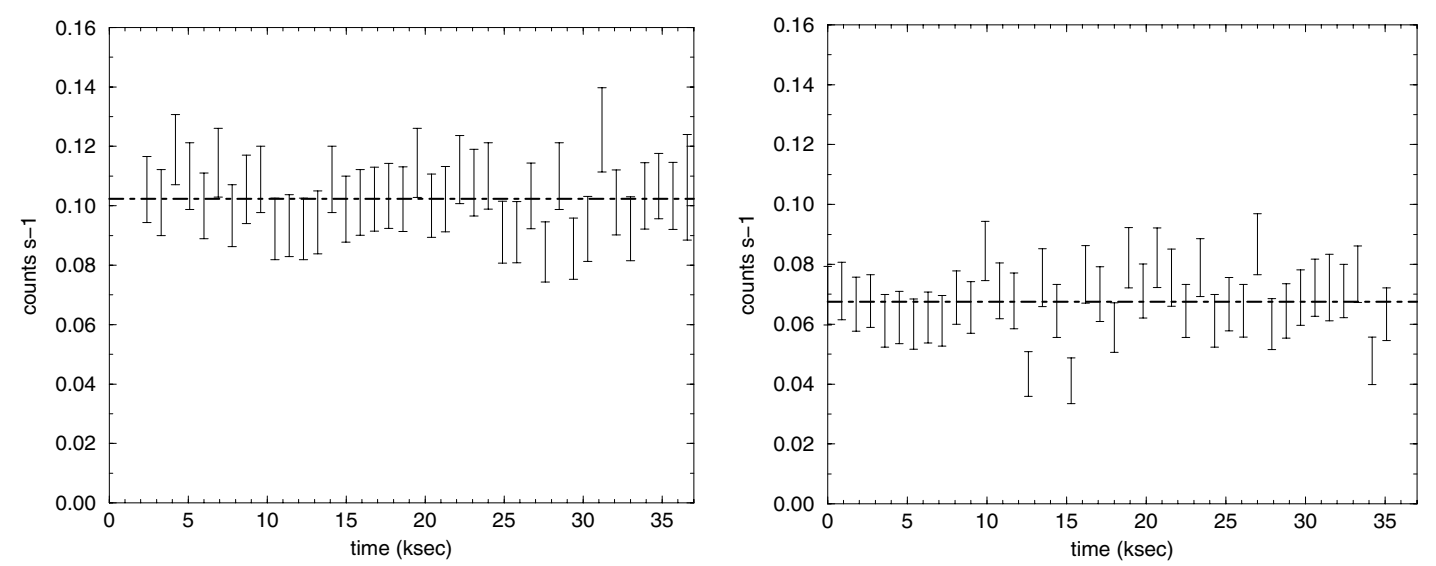

Fig. 1. Light curves of NGC 3226 obtained with the EPIC p-n (left) and the EPIC MOS (right) cameras in the $0.3-10 \mathrm{keV}$ band. The events are binned into $900 \mathrm{~s}$ time intervals. The error bars give the $1 \sigma$ count rate errors of each individual bin.

to reject visible light. The raw event data sets were processed with the "emproc" and "epproc" pipeline tasks of the XMM-Newton Science Analysis System (SAS version 5.2.0) in order to generate calibrated event lists. The source spectra of the NGC 3226 nucleus in the EPIC MOS cameras were built from photons detected within a circle of $34^{\prime \prime}$ radius. The PN spectra were extracted from a window of only $20^{\prime \prime}$ radius due to the presence of a CCD edge. These extraction windows include two X-ray sources CXOU J102334.1+195347 and CXOU102326.7+195407 detected by the Chandra observatory. However, their fluxes (a few times $10^{-14} \mathrm{erg} \mathrm{cm}^{-2} \mathrm{~s}^{-1}$ in the $2-10 \mathrm{keV}$ band; George et al. 2001) are much weaker than that of the NGC 3226 nucleus (see Sect. 3). The background was estimated on the same CCD chips within windows of similar size which were offset from the source position in a region devoid of sources. Background rates were low during the whole observation. The Pulse-Invariant (PI) spectra were rebinned so that each resultant channel had at least 40 counts per bin in the p-n spectra and 20 counts per bin in the MOS spectra. $\chi^{2}$ minimization was used for the spectral fitting. All fits were performed using the XSPEC package (v11). We used EPIC response matrices provided by the PI institutes.

\section{Integrated flux and temporal behavior}

The spectral analysis of the NGC 3226 data (see Sect. 4) yields flux measurements in the low $(0.4-2 \mathrm{keV})$ and high $(2-10 \mathrm{keV})$ energy bands of $F_{\mathrm{LE}}=1.52_{-0.04}^{+0.01} \times$ $10^{-13} \mathrm{erg} \mathrm{cm}^{-2} \mathrm{~s}^{-1}$ and $F_{\mathrm{HE}}=6.38_{-0.10}^{+0.13} \times 10^{-13} \mathrm{erg} \mathrm{cm}^{-2} \mathrm{~s}^{-1}$, respectively after correction of the Galactic absorption by the hydrogen column density $\left(N_{\mathrm{H}}=2.08 \times 10^{20} \mathrm{~cm}^{-2}\right.$; cf. Murphy et al. 1996). These correspond to luminosities of $L_{\mathrm{LE}}=$ $0.57_{-0.02}^{+0.01} \times 10^{40} \mathrm{erg} \mathrm{s}^{-1}$ and $L_{\mathrm{HE}}=2.38_{-0.04}^{+0.05} \times 10^{40} \mathrm{erg} \mathrm{s}^{-1}$ for $z=0.00441$ (de Vaucouleurs et al. 1991) and $H_{0}=$ $75 \mathrm{~km} \mathrm{~s}^{-1} \mathrm{Mpc}^{-1}$. X-ray emission from the nuclear region of NGC 3226 has been detected previously with the ROSAT PSPC (Komossa \& Fink 1997; Sansom et al. 2000) with a flux of $4.48 \pm 0.824 \times 10^{-13} \mathrm{erg} \mathrm{cm}^{-2} \mathrm{~s}^{-1}$ which implies a luminosity $L_{0.1-2 \mathrm{keV}} \approx 10^{40} \mathrm{erg} \mathrm{s}^{-1}$ for $H_{0}=75 \mathrm{~km} \mathrm{~s}^{-1} \mathrm{Mpc}^{-1}$. Chandra observations performed in 1999 December provided luminosities corrected for absorption of $1.7_{-1.3}^{+2.2} \times 10^{40} h_{75}^{-1} \mathrm{erg} \mathrm{s}^{-1}$ and
$1.8_{-0.3}^{+0.8} \times 10^{40} h_{75}^{-1} \mathrm{erg} \mathrm{s}^{-1}$ in the $0.4-2 \mathrm{keV}$ and $2-10 \mathrm{keV}$ energy bands respectively (George et al. 2001). The fluxes measured by Chandra in December 1999 and XMM-Newton in November 2000 are therefore comparable both in the low and high energy bands. There is no evidence for an X-ray luminosity variation between the two observations on the time scale of a year. The light curves for NGC 3226 (see Fig. 1) do not show significant flux variations in the $0.3-10 \mathrm{keV}$ band over the $37 \mathrm{ks}$ observation period. The EPIC pn light curve (see Fig. 1 left) is made of 39 bins of $900 \mathrm{~s}$, each containing about 90 counts on average. A constant count rate proves to be an excellent fit to the light curve. A $\chi^{2}$ value of 23.9 is obtained. The probability that a random variable exceeds this value is higher than $96 \%$. No flux variation was detected on a time scale of hours either in the $0.3-2.0 \mathrm{keV}$ or $2.0-10.0 \mathrm{keV}$ energy bands.

\section{Spectral analysis}

In order to parameterize the high energy spectrum of NGC 3226, we first consider a model using the XSPEC package in which a single power-law continuum is absorbed by the Galactic column density $N_{\mathrm{H}}=2.08 \times 10^{20} \mathrm{~cm}^{-2}$. A statistically acceptable fit to the $3-10 \mathrm{keV}$ EPIC MOS and pn data excluding the 5-7 keV iron K-shell region of NGC 3226 spectrum is obtained with a photon index $\Gamma=1.96 \pm 0.12$. The best fit parameters are given in Table 1 (see Model A). In order to check for the presence of an $\mathrm{Fe} \mathrm{K}$ emission line, we fitted the EPIC data in the 3-10 keV energy range with a phenomenological model consisting of an absorbed power law continuum and a Gaussian emission line. In the absence of any best fit line energy, we froze the energy of the Gaussian line to the position of the iron $\mathrm{K} \alpha$ fluorescence line at $6.4 \mathrm{keV}$. The addition of the Gaussian line to the spectral model does not lead to a significant change in $\chi^{2}$ fit statistics $\left(\Delta \chi^{2} / \Delta v=0.37\right.$ for 124 degrees of freedom). The best fit Gaussian line model to the line at $6.4 \mathrm{keV}$ (see Table 1, Model B) provides an upper limit to the $\mathrm{Fe} \mathrm{K} \alpha$ fluorescence line equivalent width of $17 \mathrm{eV}$.

Below $2.5 \mathrm{keV}$, a simultaneous fit of the EPIC MOS and p-n data with a power law spectrum indicates a strong absorption. A simple power law model with, cold, uniform, solar abundance absorption provides an unacceptable fit to the data. 
Table 1. Phenomenological models of the NGC 3226 EPIC spectra in the $3-10 \mathrm{keV}$ spectral band. Spectral fitting with model A does not include the 5-7 keV energy range.

\begin{tabular}{cccc}
\hline \hline Component & Parameters & Model A & Model B \\
\hline WABS & $N_{\mathrm{H}, 0}^{\mathrm{gal}}\left(\mathrm{cm}^{-2}\right)$ & $2.08 \times 10^{20}$ & $2.08 \times 10^{20}$ \\
ZPOWERLW & $\Gamma$ & $1.96 \pm 0.11$ & $1.86 \pm 0.09$ \\
& Norm & $(2.5 \pm 0.8) \times 10^{-4}$ & $(2.2 \pm 0.6) \times 10^{-4}$ \\
\hline \multirow{3}{*}{ ZGAUSS } & $E(\mathrm{keV})$ & 6.4 (frozen $)$ \\
& $\sigma(\mathrm{eV})$ & 0.05 (frozen) \\
& $E W(\mathrm{eV})$ & $17 \pm 1$ \\
\hline & $\chi_{v}^{2}$ & $0.93(84 / 90$ d.o.f.) & $0.92(114 / 124$ d.o.f.) \\
\hline
\end{tabular}

Table 2. Best fit parameters to the spectrum of NGC 3226 in the 0.3 to $10 \mathrm{keV}$ range using either a partial covering fraction absorption by neutral matter alone (Model A) or in combination with an ionized absorber model (Model B) developed by Magdziarz \& Zdziarski (1995). The best fit parameters to a thermal bremsstrahlung emission model absorbed by neutral material are also provided (Model C).

\begin{tabular}{ccccc}
\hline \hline Component & Parameters & Model A & Model B & Model C \\
\hline WABS & $N_{\mathrm{H}}\left(\mathrm{cm}^{-2}\right)($ frozen $)$ & $2.08 \times 10^{20}($ frozen $)$ & $2.08 \times 10^{20}($ frozen $)$ & $2.08 \times 10^{20}($ frozen $)$ \\
\hline ZPCFABS & $N_{\mathrm{H}, z}^{\mathrm{neu}}\left(\mathrm{cm}^{-2}\right)$ & $(7.9 \pm 0.2) \times 10^{21}$ & $(4.1 \pm 0.9) \times 10^{21}$ & $(5.3 \pm 0.4) \times 10^{21}$ \\
& Covering frac. & $\left(95_{-10}^{+5}\right) \%$ & $95 \%($ frozen $)$ & $(91 \pm 1) \%$ \\
\hline \multirow{5}{*}{ ABS. Model } & $N_{\mathrm{W}}\left(\mathrm{cm}^{-2}\right)$ & $(5.1 \pm 1.0) \times 10^{21}$ & \\
& Temperature $(\mathrm{K})$ & $3 \times 10^{4}($ frozen $)$ & \\
& $\xi\left(\mathrm{erg} \mathrm{s}^{-1} \mathrm{~cm}\right)$ & $4.1 \pm 2.9$ & \\
\hline ZPOWERLW & $\Gamma$ & 1.96 & 1.96 & $9.1 \pm 0.9$ \\
\hline ZBREMSS & $k T$ & $1.02(397 / 388$ d.o.f. $)$ & $0.99(383 / 387$ d.o.f. $)$ & $0.92(356 / 387$ d.o.f. $)$ \\
\hline
\end{tabular}

Hence, we fitted the EPIC MOS and pn spectra of NGC 3226 between 0.3 and $10 \mathrm{keV}$ with a phenomenological model consisting of (i) a red-shifted power law representing the primary continuum; (ii) Galactic absorption fixed at the level determined by the $\mathrm{H} \mathrm{I} 21 \mathrm{~cm}$ measurements of $2.08 \times 10^{20} \mathrm{~cm}^{-2}$ and (iii) a partial covering fraction absorption by neutral matter with cosmic abundance in the rest frame of the source. The photon index of the power law continuum was frozen to the value derived from the best fit power law models to the EPIC spectra above $3 \mathrm{keV}$ (see Table 1, Model A). Absorption with a partial covering fraction included between 85 and $100 \%$ provides an acceptable fit to the data $\left(\chi_{v}^{2}=1.02\right.$; see Table 2 Model A) using the absorption cross-sections from Balucinska-Church \& McCammon (1992) with a He cross-section based on Yan et al. (1998). The best fit model indicates the presence of a large column density of cold material $N_{\mathrm{H}}=(7.9 \pm 0.2) \times 10^{21} \mathrm{~cm}^{-2}$ in the line of sight to the nucleus.

In order to detect a possible absorption contribution from ionized matter, we then fitted the EPIC spectra in the $0.3-10 \mathrm{keV}$ range with a power-law model absorbed by neutral and ionized material. We used the ionized absorber model ABSORI (in XSPEC) to quantify the column density of the ionized plasma, $N_{\mathrm{W}}$, and the ionization parameter, $\xi$, of the ionized absorber. In this single zone equilibrium ionization model the opacity of the gas is based on the ionization distribution of the relevant atomic species in a slab of Thomson-thin plasma as a function of the ionization parameter. This parameter is defined as $\xi=L / n R^{2}$, where $n$ is the number density of the warm plasma and $R$ the distance from the ionizing source with an isotropic luminosity $L$ in the interval $5 \mathrm{eV}$ to $20 \mathrm{keV}$. The photon index of the incident power law continuum was frozen to the value derived from the best fit power law model to the EPIC spectra above $3 \mathrm{keV}$ (see Table 1; Model A). The results of the spectral fitting using a power law absorbed by neutral and ionized matter are given in Table 2. The best fit parameters $\left(\chi_{v}^{2} \approx 0.99\right)$ to the EPIC spectra of NGC 3226 using a single zone warm absorber model give a low ionization parameter $\xi=4 \pm 3 \mathrm{erg} \mathrm{s}^{-1} \mathrm{~cm}$ and a hydrogen column density $N_{\mathrm{W}}=(5 \pm 1) \times 10^{21} \mathrm{~cm}^{-2}$ for the ionized plasma. According to Kallman \& Bautista (2001), the temperature of an optically photoionized gas in thermal equilibrium with a ionization parameter $\xi=10 \mathrm{erg} \mathrm{s}^{-1} \mathrm{~cm}$ is around $4 \times 10^{4} \mathrm{~K}$ with only a small dependence on density over a wide range included between $10^{6} \mathrm{~cm}^{-3}$ to $10^{16} \mathrm{~cm}^{-3}$. Such a temperature leads to an acceptable description of the spectrum of NGC 3226. The improvement in $\chi^{2}$ fit statistics by adding a single zone absorber 

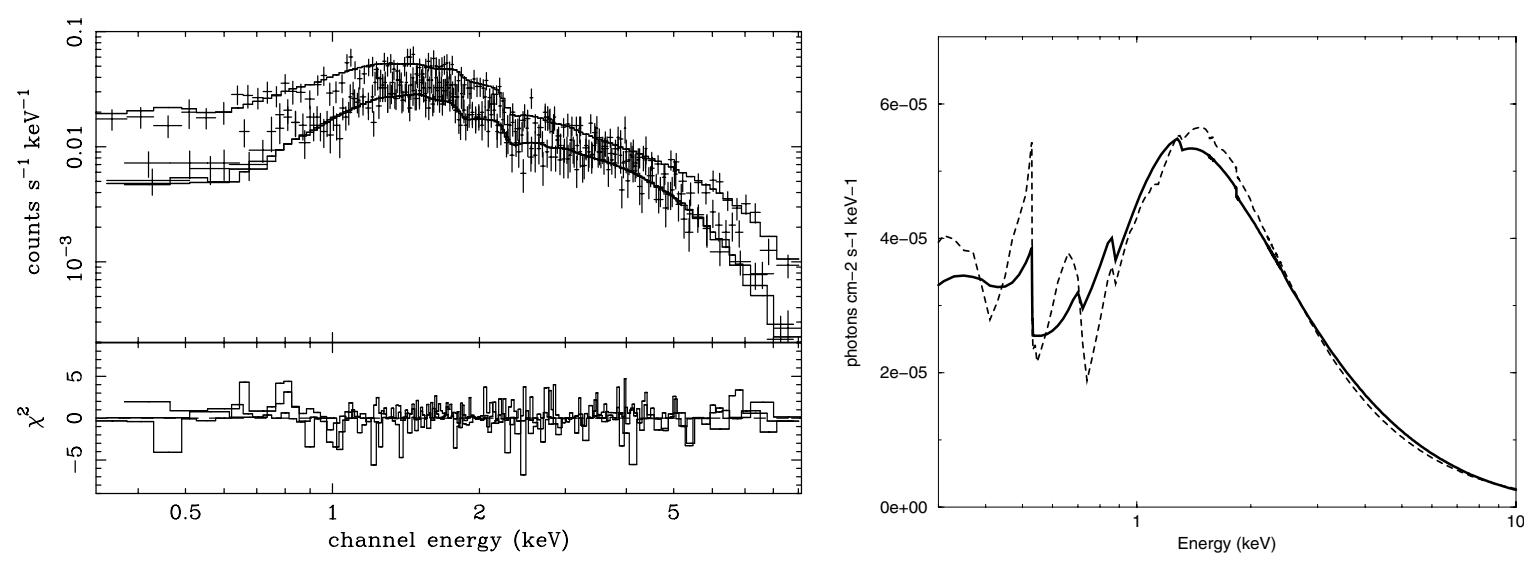

Fig. 2. Left: simultaneous fit (see Table 2) of the EPIC pn, EPIC MOS 1 and EPIC MOS 2 spectra by a bremsstrahlung model absorbed by neutral gas. The $\chi^{2}$ distribution is displayed in the lower panel. The corresponding spectral model (bold line) is plotted on the right panel and compared with the best fit absorbed power law model (dashed line) described in Table 2 (Model B).

model $\left(\Delta \chi^{2} / \Delta v=14\right.$ for 387 degrees of freedom) is significant at $>99.9 \%$ confidence using the F-statistic. However, we found that the X-ray spectrum of NGC 3226 is also consistent $\left(\chi_{v}^{2}=0.92\right)$ with a thermal bremsstrahlung emission with $k T=(9.1 \pm 0.9) \mathrm{keV}$ absorbed by a column density $N_{\mathrm{H}, z}^{\text {neu }}=(5.3 \pm 0.4) \times 10^{21} \mathrm{~cm}^{-2}$ of neutral material with a covering fraction equal to $91 \pm 1 \%$ (see Table 2; Model C). The improvement in $\chi^{2}$ fit statistics of the absorbed bremsstrahlung model over the absorbed power law model $\left(\Delta \chi^{2} / \Delta v=41\right.$ for 387 degrees of freedom) is even larger than after adding a weakly ionized absorber to the power law model. Hence, we conclude that the bremsstrahlung model absorbed by neutral material provides the best description of the X-ray spectrum of NGC 3226 in the $0.3-10 \mathrm{keV}$ range (see Fig. 2).

\section{Discussion}

The analysis results of the XMM-Newton observation of NGC 3226 show that the spectrum of its central region above $3 \mathrm{keV}$ can be described by a power law continuum with $\Gamma \approx$ 1.96. The continuum X-ray emission of NGC 3226 at high energies looks therefore similar to that of Seyfert 1 galaxies ( $\Gamma \approx 1.9$; Nandra \& Pounds 1994 ), consistent with the findings of a previous observation of the source by Chandra (George et al. 2001). However, the X-ray luminosity of NGC 3226 is about 2-3 orders of magnitude lower than typical Seyfert galaxies as expected from the presence of a low luminosity active galactic nucleus in a LINER (Ho 1999; Terashima et al. 2000). The spectrum of NGC 3226 nucleus is absorbed at low energies by neutral gas with a hydrogen column density $N_{\mathrm{H}} \approx(3-10) \times 10^{21} \mathrm{~cm}^{-2}$ and a covering fraction greater than $85 \%$. This value is comparable with the equivalent hydrogen column density $N_{\mathrm{H}}=4.8_{-1.5}^{+1.7} \times 10^{21} \mathrm{~cm}^{-2}$ reported by George et al. (2001) which corresponds to $2 \leq A_{v} \leq 4$ assuming a Galactic dust to gas ratio. This is consistent with the fact that no significant luminosity variation is detected between the Chandra observation in December 1999 and the XMM-Newton observation in November 2000. A fraction of the $\mathrm{X}$-ray continuum emitted by the nucleus could be attenuated due to the presence of ionized material intrinsic to NGC 3226.
A possible spectral model of the source suggests the presence of weakly ionized gas $\left(N_{\mathrm{W}} \approx(4-6) \times 10^{21} \mathrm{~cm}^{-2}\right.$ with $\xi \approx 1-7 \mathrm{erg} \mathrm{s}^{-1} \mathrm{~cm}$ ) in the nucleus of NGC 3226. Assuming that NGC 3226 is a genuine AGN and using a primary continuum with a power law index $\Gamma=1.96$ (see Table 1 ), we calculated a ionizing luminosity $L_{\text {ion }}=9.86 \times 10^{40} \mathrm{erg} \mathrm{s}^{-1}$ in the 1-1000 Rydberg range for a distance of $17.6 \mathrm{Mpc}(z=0.00441$ and $\left.H_{0}=75 \mathrm{~km} \mathrm{~s}^{-1} \mathrm{Mpc}^{-1}\right)$. Under the assumption that the species involved are in ionization balance, this leads to an estimate of the maximum distance of the ionized absorbing region $r_{\text {max }} \approx 30 \times L_{\text {ion }, 44} \times N_{22}^{-1} \times \xi_{100}^{-1}$ pc (Krolik \& Kriss 2001), i.e. $r_{\text {max }}=0.7-6.0$ pc for $L_{\text {ion }, 44} \approx 9.86 \times 10^{-4}, N_{22}=0.4-0.6$ and $\xi_{100}=0.01-0.07$ where the fiducial quantities $\left(10^{44} \mathrm{erg} \mathrm{s}^{-1}\right.$ for $L_{\text {ion }}, 10^{22} \mathrm{~cm}^{-2}$ for $N$ and 100 for $\xi$ ) are representative of those commonly inferred for AGNs. Although consistent with the classification of NGC 3226 as a LINER, a model assuming the presence of neutral and weakly ionized gas absorbing a power law continuum does not provide the best fit to the EPIC spectra of NGC 3226 nucleus.

Our analysis indicates that a bremsstrahlung model absorbed by neutral material is a significantly better description. This result is reminiscent of the X-ray emission from advection-dominated accretion flows which is believed to be dominated by thermal bremsstrahlung. On account of their relatively low X-ray luminosities and hard X-ray emission, LINERS are prime candidates for ADAFs (Yi \& Boughn 2001). The optical luminosity of the entire galaxy NGC $3226\left(M_{\mathrm{B}}=\right.$ $\left.-19.4 ; L_{\mathrm{B}} / L_{\odot} \approx 8 \times 10^{9}\right)$ and a central line of sight dispersion $\left(\approx 180 \mathrm{~km} \mathrm{~s}^{-1}\right.$; Simien \& Prugniel 1998) are consistent with a relation for a variety of galaxies harboring black holes (e.g. Gebhardt et al. 2000 and references therein). The implied central black hole mass is $\approx 10^{8} M_{\odot}$ and the accretion rate is most likely lower than a percent of the Eddington accretion rate (George et al. 2001). Narayan \& Yi (1995) found that advection-dominated flows occur for mass accretion rates of typically a hundredth or a thousandth of the Eddington rate. When the density of the accretion flow is sufficiently low, the radiative cooling rate becomes smaller than the viscous heating rate. As a result, the dissipated accretion energy 
is not efficiently radiated away but kept as internal heat and advected inward with the accreted plasma (Rees et al. 1982). One interesting result of the study conducted by Narayan \& Yi (1995) is that the electron temperature at the accretion rate below which advection-dominated accretion occurs does not vary much for a whole range of black hole masses. The bremsstrahlung emission has similar characteristics and accretion driven black holes reach typical electron temperatures of $T_{\mathrm{e}} \approx 10^{9}-10^{9.5} \mathrm{~K}$ at small radii from the central object. The temperature of the best fit bremsstrahlung model to the EPIC spectra of NGC 3226 is about one order of magnitude lower than this value $\left(T \approx 10^{8} \mathrm{~K}\right)$. This temperature and the absence of short and mid time-scale variability between the $X M M-N e w t o n$ and the Chandra observations suggest that the X-ray emission originates from regions at a relatively large distance from the central object (i.e. greater than a few thousand Schwarschild radii) where the dynamical time-scale is months to years as in the so-called wind models (Blandford \& Begelman 1999; Di Matteo et al. 2000). It is worth noting that the ADAF interpretation is supported by the absence of an Fe K $\alpha$ line and by the low X-ray luminosity of the NGC 3226 nucleus. However, numerical simulations have shown that such flows should be convectively unstable (e.g. Igumenshev et al. 2001) and that convection is strong whenever the viscosity parameter is small. Such convection dominated accretion flows (CDAFs) have a different structure than ADAFs (Stone et al. 1999). The angular momentum that convection transports inwards nearly cancels the normal outward transport by viscosity (Narayan et al. 2000; Quatert \& Gruzinov 2000). As a result, a nearly static accretion flow is established in which most of the gas circulates in convective eddies rather than accreting onto the central object. For fixed boundary conditions at large radii, the mass accretion rate in a CDAF is thus much smaller than in a nonconvecting ADAF. Convection in CDAFs transports the luminosity from small to large radii. The energy is supplied by the small amount of mass accreting onto the black hole but a significant fraction of this energy may be radiated from the outer regions of the CDAF as thermal bremstrahlung emission (Narayan et al. 2000; Igumenshev \& Abramowicz 2001). Ball et al. (2001) showed that this leads to a correlation between the frequency of maximum bremstrahlung emission and the luminosity of the source, $v_{\text {peak }} \approx L^{2 / 3}$. Accreting black holes with X-ray luminosities $10^{-4} L_{\text {Edd }} \geq L_{0.5-10 \mathrm{keV}} \geq 10^{-7} L_{\text {Edd }}$ are expected to have hard X-ray spectra, with photon indices $\Gamma \approx 2$, similar to the X-ray spectrum of NGC 3226. Hence, although ADAFs are the simplest dynamic models of a radiatively inefficient flow from which spectral energy distribution can be derived, radiatively inefficient flows may be significantly modified by convection or outflows. Abramovicz et al. (2002) showed that radiatively inefficient accretion flows with low viscosity actually consists of both an outer convectiondominated zone and an inner advection dominated zone.

Assuming that the hard $\mathrm{X}$-ray emission is from a radiatively inefficient ADAF around a massive black hole, Yi \& Boughn (2001) showed that $\mathrm{X}$-ray luminosity and radio luminosity satisfy the approximate relation $L_{\mathrm{R}} \approx 7 \times 10^{35}(v / 15 \mathrm{GHz})^{7 / 5}$ $\left(M / 10^{7} M_{\odot}\right)\left(L_{\mathrm{X}} / 10^{40} \mathrm{erg} \mathrm{s}^{-1}\right)^{1 / 10}$ where $L_{\mathrm{R}}=v L_{v}$ is the radio luminosity at frequency $v, M$ is the mass of the accreting black hole, and $L_{\mathrm{X}}$ the luminosity in the $2-10 \mathrm{keV}$ range. Using $L_{\mathrm{X}}=2.58 \times 10^{40} \mathrm{erg} \mathrm{s}^{-1}$ after correction from galactic and intrinsic absorption and flux densities of 3.5-4.8 mJy and $5.0 \mathrm{mJy}$ at $5 \mathrm{GHz}$ and $15 \mathrm{GHz}$ respectively (Falcke et al. 2000), we find $M=(3-5) \times 10^{8} M_{\odot}$. Recently, Merloni et al. (2003) have performed a partial correlation analysis on a broad sample of about 150 supermassive and galactic stellar mass blackholes, and also concluded that the X-ray luminosity correlates with both mass and radio luminosity. They found that the sources lie preferentially on a plane in a three dimensional $\left(\log L_{\mathrm{R}}, \log L_{\mathrm{X}}, \log M\right)$ space, described by the equation $\log$ $L_{\mathrm{R}}=0.6_{-0.11}^{+0.11} \log L_{\mathrm{X}}+0.78_{-0.09}^{+0.11} \log M+7.33_{-4.07}^{+4.05}$ with however a significant scatter. When applied to NGC 3226, a mass $M=1.7 \times 10^{7} M_{\odot}$ is obtained which is comparable with the $10^{8} M_{\odot}$ estimate from line-of-sight velocity dispersions. The existence of a correlation between X-ray and radio emission is the indication of a fundamental connection between accretion flows and jet activities and the correlation coefficients provide probes of the accretion physics and of the inner jets. It is argued (Merloni et al. 2003; Fackle et al. 2004) that black holes operating at sub-Eddington accretion rates make a transition to a radiation inefficient state where most of the emission is dominated by the non-thermal emission of a jet. This suggests that the dwarf elliptical galaxy NGC 3226 as other nearby elliptical galaxies (Di Matteo et al. 2000) may harbor a sub-Eddington super-massive black hole in a radiative inefficient state which is distinct from the near-Eddington, thermally dominated blackhole presumably found in quasars and luminous Seyfert galaxies.

Acknowledgements. We thank our colleagues from the XMM-Newton Science Operation Centers for their support in implementing the observations. We are grateful to the anonymous referee for the helpful comments that allowed to improve the paper.

\section{References}

Abramovicz, M. A., Igumenshchev, I. V., Quataert, E., \& Narayan, R. 2002, ApJ, 565, 1101

Ball, G. H., Narayan, R., \& Quataert, E. 2001, ApJ, 552, 221

Balucinska-Church, M., \& McCammon, D. 1992, ApJ, 400, 699

Blandford, R., \& Begelman, M. C. 1999, MNRAS, 303, L1

Di Matteo, T., Quataert, E., Allen, S. W., et al. 2000, MNRAS, 311, 507

Falcke, H., Nagar, N. M., Wilson, A. S., et al. 2000, ApJ, 542, 197

Falcke, H., Körding, E., \& Markoff, S. 2004, A\&A, 414, 895

Gebhardt, K., Bender, R., Bower, G., et al. 2000, ApJ, 539, L13

George, I. M., Mushotzky, R., Yaqoob, T., et al. 2001, ApJ, 559, 167

Gondoin, P., Aschenbach, B., Erd, C., et al. 2000, SPIE Proc., 4140, 1

Gondoin, P., Orr A., Lumb, D., \& Siddiqui, H. 2003, A\&A, 397, 883

Ho, L. C., Filippenko, A. V., Sargent, W. L. W., et al. 1997, ApJS, 112, 391

Ho, L. C. 1999, ApJ, 516, 672

Igumenshchev, I. V., \& Abramowicz, M. A. 2000, ApJS, 130, 463

Igumenshchev, I. V., Abramowicz, M. A., \& Narayan, R. 2000, ApJ, 537, L27

Jansen, F., Lumb, D., Altieri, B., et al. 2001, A\&A, 365, L1

Kallman, T., \& Bautista, M. 2001, ApJS, 133, 221

Komossa, S., \& Fink, H. 1997, A\&A, 327, 483

Krolik, J. H., \& Kriss, G. A. 2001, ApJ, 561, 684 
Magdziarz, P., \& Zdziarski, A. 1995, MNRAS, 273, 837

Merloni, A., Heinz, S., \& Di Matteo, T. 2003, MNRAS, 345, 1057

Murphy, E. M., Lockman, F. J., Laor, A., \& Elvis, M. 1996, ApJS, 105,369

Nandra, K., \& Pounds, K. A. 1994, MNRAS, 268, 405

Narayan, R., \& Yi, I. 2000, ApJ, 452, 710

Narayan, R., Igumenshchev, I. V., \& Abramowicz, M. A. 2000, ApJ, 539, 798

Quataert, E., \& Narayan, R. 1999, ApJ, 550, 298

Quataert, E., \& Gruzinov, A. 2000, ApJ 539, 809

Rees, M. J., Begelman, M. C., Blandford, R. D., et al. 1982, Nature, 295,17

Sansom, A. E., Hibbard, J. E., \& Schweizer, F. 2000, AJ, 120, 1946
Simien, F., \& Prugniel, P. 1998, A\&AS, 131, 287

Stone, J. M., Pringle, J. E., \& Begelman, M. C. 1999, MNRAS, 310, 1002

Strüder, L., Briel, U., Dennerl, K., et al. 2001, A\&A, 365, L18

Terashima, Y., Ho, L. C., \& Ptak, A. F. 2000, ApJ, 539, 161

Turner, M. J. L. T., Abbey, A., Arnaud, M., et al. 2001, A\&A, 365, L27

de Vaucouleurs, G., de Vaucouleurs, A., Corwin, Jr. H. G., et al. 1991, Third Refeence Catalogue of Bright Galaxies version RC3.9 (New-York: Springer)

Yan, M., Sadeghpour, H. R., \& Dalgarno, A. 1998, ApJ, 496, 1044 Yi, I., \& Boughn, S. P. 1998, ApJ, 499, 198 